\title{
PRICING EXOTIC OPTION UNDER STOCHASTIC VOLATILITY MODEL
}

\author{
Pengshi Li
}

\section{Introduction}

Exotic options are called "customer tailored options" or "special purpose option" because they are flexible to be tailored to the specific needs of investors. Strategies based on exotic options are often employed to hedge the specific risk exposures from the financial markets. Because exotic options are more efficient and less expensive than their standard counterparts, they are playing a significant hedging role in cost effective ways. Unlike the vanilla call and put options, exotic options are either variation on the payoff patterns of plain vanilla options or they are totally different kinds of derivatives with other features. While simple vanilla call and put options are traded in the exchanges, most of the exotic options are traded in the over-the-counter markets.

Supershare option and chooser option are two typical kinds of exotic options which suggest a broad range of usage and application in different financial market conditions. Supershare option is one type of binary option. Unlike the binary option which has only one boundary, a supershare option has an upper and lower boundary. If the underlying asset price is between these boundaries, the payoff of the supershare option is the ratio of the underlying asset price and the lower boundary, otherwise the supershare option is worthless. Chooser option gives the holder the right to choose at some future date before maturity whether the option will finally be a put or a call with a common exercise price. Chooser options are suitable for investors who are certain about a strong volatility of the underlying asset price but uncertain about the direction of the change.

Exact analytical formulae for supershare option and chooser option can be derived through the Black-Scholes world. Despite the popularity and longevity of the Black-
Scholes model, the assumption of constant volatility in the Black-Scholes model contradicts to the existence of the non-flat implied volatility surface observed in empirical studies. Bernales and Guidolin (2015) prove the existence of predictability patterns in the movement of implied volatility surface. Various methods are hence proposed to remedy the unrealistic constant volatility assumption, one of them is the stochastic volatility (SV) model which is suitable for the medium and long term maturity options.

A typical stochastic volatility model assumes the volatility is driven by a meanreverting diffusion process. The motivation of this assumption is that empirical researches find evidences of mean-reverting future, which was not taken into account in the traditional option pricing model, in many financial assets such as stock price; see Alizadeh et al. (2002). Another motivation of the stochastic volatility assumption is that by considering an instant correlation between the standard Brownian motions in the process driving the underlying asset price and the process driving the volatility, the stochastic volatility model can explain the leverage effect observed in financial markets. Smith (2015) finds the leverage effects in the U.S. stock markets. The leverage effect suggests volatility shocks are negatively correlated with underlying asset price shock, it also accounts for a skewed distribution for the asset price observed by Carmichael and Coen (2013).

Studies on option pricing under stochastic volatility model had been carried out for years. Heston (1993) obtained a closed-form formula for options on bonds and currency in terms of characteristic functions; Fouque et al. (2003) performed singular perturbation analysis on the fast-mean reverting stochastic volatility model. And the accuracy of the corresponding 
approximation in the presence of the nonsmoothness of payoff functions (such as call option) is also studied; Their asymptotic option pricing method has been applied to the research of turbo warrants pricing by Wong et al. (2008); Park and Kim (2013) investigate a semi-analytic pricing method for lookback options in a general stochastic volatility framework; Yang et al. (2014) derive an analytic approximation formula for the price of the vulnerable options when the underlying asset follows a stochastic volatility; Zhu and Lian (2015) work out analytical closed-form formulae for the price of forward-start variance swap based on the Heston stochastic volatility model; Cao et al. (2016) investigate the pricing problem of variance swaps under stochastic volatility and stochastic interest rate; Lee et al. (2016) obtain a closed-form analytic formula of the price of the European vulnerable option assuming that the underlying asset price follows the Heston dynamics. Although many studies are devoted to option pricing under stochastic volatility model in recent years, to the best of our knowledge, research on exotic option such as supershare and chooser option pricing have not been carried out in the stochastic volatility case.

By extending the constant volatility in BlackScholes model, this paper studies the pricing of the supershare option and chooser option in a fast mean-reverting stochastic volatility scenario. This work may contribute to the exotic option pricing literature in several ways. Supershare and chooser options are both important financial instruments, research on these two exotic options in stochastic volatility model may give more insights on the pricing of supershare and chooser options. Further, this paper presents the semi-analytical formulae of the supershare and chooser option. These formulae are critical to the determination of option delta and hedge ratio of the replicate portfolio which is important to investors who use these exotic options to hedge their risk exposure.

The rest of this paper is organized as follows. Section 1 formulate the model setting of the underlying stochastic volatility model; Section 2 derives the zero-order and first-order approximation of the supershare option and chooser option; A numerical result of these two exotic options are carried out in Section 3; And the final section concludes this paper.

\section{Research Methodology}

Denoting $S_{t}$ as the price of the underlying asset; the driving volatility $Y_{t}$ follows an $\mathrm{O}-\mathrm{U}$ process under real world probability measure $P$. The stochastic volatility model considered in this section is described by five parameters which are: $m$ the long run mean level of the driving volatility $Y_{t} ; \alpha$ the mean-reverting rate of $Y_{t} ; v$ the standard deviation of the invariant distribution of $Y_{t} ; \rho$ the instant correlation captures the skewness, and $\gamma$ the market price of the volatility risk. Assumes that the risk-free interest rate $r$ is constant, the market selects a constant market price of volatility risk and price derivatives under a risk-neutral world probability measure $P^{*}$, then the stochastic volatility model under such probability measure can be written as:

$$
\begin{aligned}
& d S_{t}=r S_{t} d t+f\left(Y_{t}\right) S_{t} d W_{t}^{*} \\
& d Y_{t}=\left[\alpha\left(m-Y_{t}\right)-v \sqrt{2 \alpha} \Lambda\left(Y_{t}\right)\right] d t+ \\
& +v \sqrt{2 \alpha}\left(\rho d W_{t}^{*}+\sqrt{1-\rho^{2}} d Z_{t}^{*}\right) \\
& \Lambda(y)=\rho \frac{(\mu-r)}{f(y)}+\gamma \sqrt{1-\rho^{2}}
\end{aligned}
$$

where $f(y)$ is assumed to be a positive, bounded and sufficiently regular function. $W_{t}^{*}$ and $Z_{t}^{*}$ are two independent standard Brownian motions under risk-neutral world probability measure $P^{*}$. $Y$ has a invariant normal distribution with mean $m$ and variance $v^{2} . \Lambda(y)$ is defined as the combined market price of risk.

Consider a European exotic option with an expiry date $T$ and payoff $g(s)$ at expiry date. Since under the risk-neutral world probability measure $P^{*}$, the processes $\left(S_{t}, Y_{t}\right)$ is a Markov process, the price of this European exotic option at time $t<T$ is a function of the present value of the underlying asset price and the present value of the process driving the volatility. Then the price of the European exotic option, which denote as $V(t, s, y)$, at time $t$ is given by:

$$
\begin{aligned}
& V(t, s, y)=\mathrm{E}^{*}\left[e^{-r(T-t)} g\left(S_{T}\right) \mid\right. \\
& \left.\mid S_{t}=s, Y_{t}=y\right]
\end{aligned}
$$


It can also be obtained as the solution of the following partial differential equation by Feyman-Kac formula:

$$
\begin{aligned}
& \frac{\partial V}{\partial t}+r\left(s \frac{\partial V}{\partial s}-V\right)+\frac{1}{2} f^{2}(y) s^{2} \frac{\partial^{2} V}{\partial s^{2}}+ \\
& +\frac{1}{\varepsilon}\left[(m-y) \frac{\partial V}{\partial y}+v^{2} \frac{\partial^{2} V}{\partial y^{2}}\right] \\
& +\frac{1}{\sqrt{\varepsilon}}\left[v \sqrt{2} \rho f(y) s \frac{\partial^{2} V}{\partial s \partial y}-v \sqrt{2} \Lambda(y) \frac{\partial V}{\partial y}\right]=0 \\
& V(T, s, y)=g(s)
\end{aligned}
$$

where $\varepsilon$ is a small parameter and we define $\alpha=1 / \varepsilon$.

When the mean-reverting rate $\alpha$ is large, i.e. $0<\varepsilon \leq 1$. The price of the European exotic option satisfying a problem of and can be solved in asymptotic expansions which was suggested by Fouque et al. (2003). The main results of their research are presented as follow.

Result 1 The zero-order approximation $V_{0}(t, s)$ for the price of the European option in the fast-mean reverting stochastic volatility economy is independent of the present volatility and it is the standard Black-Scholes price at a constant volatility $\bar{\sigma}$ defined as below:

$$
\begin{gathered}
\frac{\partial V_{0}}{\partial t}+\frac{1}{2} \bar{\sigma}^{2} s^{2} \frac{\partial^{2} V_{0}}{\partial s^{2}}+r s \frac{\partial V_{0}}{\partial s}-r V_{0} \\
V_{0}(T, s)=g(s) \\
\bar{\sigma}^{2}=\int_{-\infty}^{+\infty} f^{2}(y) \frac{1}{\sqrt{2 \pi}} e^{-\frac{(y-m)^{2}}{2 v^{2}}} d y
\end{gathered}
$$

Result 2 The first-order approximation $\sqrt{\varepsilon} V_{1}(t, s)$ for the price of the European option in the fast-mean reverting stochastic volatility economy is independent of the present volatility and it is explicitly given as below:

$$
\begin{aligned}
& \sqrt{\varepsilon} V_{1}(t, s)=-(T-t)\left(C_{1} s^{2} \frac{\partial^{2} V_{0}}{\partial s^{2}}+\right. \\
& \left.+C_{2} s^{3} \frac{\partial^{3} V_{0}}{\partial s^{3}}\right)
\end{aligned}
$$

where $C_{1}$ and $C_{2}$ are two parameters which can be calibrated from the observed implied volatility surface.

Result 3 The price of the European option in the fast-mean reverting stochastic volatility economy can be approximated by the combination of the zero-order approximation and the first-order approximation:

$$
V=V_{0}+\sqrt{\varepsilon} V_{1}+\mathrm{O}\left(\alpha^{-1}\right)
$$

\section{Analysis}

\subsection{European Supershare Option}

The payoff of a supershare option at expiry date is defined as:

$$
S S_{T}=\frac{S_{T}}{K_{1}} 1_{\left\{K_{1}<S_{T}<K_{2}\right\}}
$$

for positive constants $K_{1}<K_{2}$. In the fast mean-reverting stochastic volatility economy, the approximate price of the supershare option is $\tilde{V}^{s}(t, s)=V_{0}^{s}(t, s)+\sqrt{\varepsilon} V_{1}^{s}(t, s)$. The first-order approximation $V_{0}^{s}(t, s)$ is simply the standard Black-Scholes option price with a constant volatility $\bar{\sigma}$.

Lemma 1 The price of the supershare option in the Black-Scholes model with constant volatility is given by:

$$
\begin{aligned}
& S S_{t}=\frac{S_{t}}{K_{1}}\left(N\left(\bar{d}_{1}\right)-N\left(\bar{d}_{2}\right)\right) \\
& \bar{d}_{1}=\frac{\ln \left(s / K_{1}\right)+\left(r+\frac{\sigma^{2}}{2}\right)(T-t)}{\sigma \sqrt{T-t}} ; \\
& \bar{d}_{2}=\frac{\ln \left(s / K_{2}\right)+\left(r+\frac{\sigma^{2}}{2}\right)(T-t)}{\sigma \sqrt{T-t}}
\end{aligned}
$$

where $K_{1}, K_{2}, r, \sigma$ are constants. $S_{t}$ is the underlying asset price at time $t, T$ is the expiry date. $N(x)$ denotes the cumulative distribution function of a standard normal probability density function.

\section{Proof of Lemma1:}

The payoff of a supershare option is defined as:

$$
S S_{T}=\frac{S_{T}}{K_{1}} 1_{\left\{K_{1}<S_{T}<K_{2}\right\}}
$$

for positive constants $K_{1}<K_{2}$. According to the risk-neutral formula the price of a supershare option at time $t$ is a function $f(t, s)$ such that: 


$$
f\left(t, S_{t}\right)=\mathrm{E}^{*}\left[e^{-r(T-t)} S S_{T} \mid S_{t}=s\right]
$$

where $W_{t}^{*}$ is a standard Brownian motion under the risk-neutral measure. Because of the property of the Brownian motion, we may write the formula above as:

where $Z$ is a standard normal random variable:

$$
Z=-\frac{W_{T}^{*}-W_{t}^{*}}{\sqrt{T-t}}
$$

Therefore:

$$
\begin{aligned}
f(t, s) & =\mathrm{E}^{*}\left[e^{-r(T-t)} \frac{s}{K_{1}} \exp \left(-\sigma Z \sqrt{T-t}+\left(r-\frac{\sigma^{2}}{2}\right)(T-t)\right) 1_{\left\{K_{1}<S_{T}<K_{2}\right\}}\right] \\
& =\int_{-\infty}^{+\infty} e^{-r(T-t)} \frac{s}{K_{1}} \exp \left(-\sigma z \sqrt{T-t}+\left(r-\frac{\sigma^{2}}{2}\right)(T-t)\right) 1_{\left\{K_{1}<S_{T}<K_{2}\right\}} \frac{1}{\sqrt{2 \pi}} e^{-\frac{z^{2}}{2}} d z
\end{aligned}
$$

Since $K_{1}<S_{T}<K_{2} \Rightarrow K_{1}<s \exp \left[-\sigma z \sqrt{T-t}+\left(r-\frac{\sigma^{2}}{2}\right)(T-t)\right]<K_{2}$, then the integrand is nonzero if and only if:

$$
d_{K_{2}}=\frac{\ln \left(s / K_{2}\right)+\left(r-\frac{\sigma^{2}}{2}\right)(T-t)}{\sigma \sqrt{T-t}}<z<d_{K_{1}}=\frac{\ln \left(s / K_{1}\right)+\left(r-\frac{\sigma^{2}}{2}\right)(T-t)}{\sigma \sqrt{T-t}}
$$

Therefore:

$$
\begin{aligned}
f(t, s) & =\int_{d_{K 2}}^{d_{K_{1}}} \frac{s}{K_{1} \sqrt{2 \pi}} \exp \left(-\frac{z^{2}}{2}-\sigma z \sqrt{T-t}-\frac{\sigma^{2}}{2}(T-t)\right) d z \\
& =\int_{d_{K 2}}^{d_{K_{1}}} \frac{s}{K_{1} \sqrt{2 \pi}} \exp \left(-\frac{1}{2}(z+\sigma \sqrt{T-t})^{2}\right) d(z+\sigma \sqrt{T-t}) \\
& =\frac{s}{K_{1}} \int_{d_{K 2}+\sigma \sqrt{T-t}}^{d_{K_{1}}+\sigma \sqrt{T-t}} \frac{1}{\sqrt{2 \pi}} e^{-\frac{t^{2}}{2}} d t=\frac{s}{K_{1}}\left(N\left(\bar{d}_{1}\right)-N\left(\bar{d}_{2}\right)\right) \\
\bar{d}_{1} & =d_{K_{1}}+\sigma \sqrt{T-t}=\frac{\ln \left(s / K_{1}\right)+\left(r+\frac{\sigma^{2}}{2}\right)(T-t)}{\sigma \sqrt{T-t}} \\
\bar{d}_{2} & =d_{K_{2}}+\sigma \sqrt{T-t}=\frac{\ln \left(s / K_{2}\right)+\left(r+\frac{\sigma^{2}}{2}\right)(T-t)}{\sigma \sqrt{T-t}}
\end{aligned}
$$




\section{Finance}

thus the price of a supershare option at time $t$ is equal to:

$$
S S_{t}=f\left(t, S_{t}\right)=\frac{S_{t}}{K_{1}}\left(N\left(\bar{d}_{1}\right)-N\left(\bar{d}_{2}\right)\right)
$$

Hence according to Result 1 in Chapter 1 and Lemma1, $V_{0}^{s}(t, s)$ is given by:

$$
\begin{aligned}
& V_{0}^{s}(t, s)=\frac{s}{K_{1}}\left(N\left(\bar{d}_{1}\right)-N\left(\bar{d}_{2}\right)\right) \\
& \bar{d}_{1}=\frac{\ln \left(s / K_{1}\right)+\left(r+\frac{\bar{\sigma}^{2}}{2}\right)(T-t)}{\bar{\sigma} \sqrt{T-t}}
\end{aligned}
$$

where $N(x)$ denotes the standard normal cumulative distribution function. Denotes $n(x)$ as the standard normal probability density function, we can easily obtain that $N^{\prime}(x)=n(x)$ and $N^{\prime}(x)=-x n(x)$. From the definition of $\bar{d}_{1}$ and $\bar{d}_{2}$, we have $\frac{\partial \bar{d}_{1}}{\partial s}=\frac{\partial \bar{d}_{2}}{\partial s}=\frac{1}{s \sigma \sqrt{T-t}}$. Hence, the second order and third order derivative of the value of the zero-order approximation with respect to the underlying asset price are given by:

$$
\begin{gathered}
\frac{\partial^{2} V_{0}^{s}}{\partial s^{2}}=\frac{1}{s K_{1} \sigma \sqrt{T-t}}\left(n\left(\bar{d}_{1}\right)-n\left(\bar{d}_{2}\right)\right)+\frac{1}{s K_{1} \sigma^{2}(T-t)}\left((-\bar{d}) n\left(\bar{d}_{1}\right)+\left(\bar{d}_{2}\right) n\left(\bar{d}_{2}\right)\right) \\
\frac{\partial^{3} V_{0}^{s}}{\partial s^{3}}=\frac{1}{s^{2} K_{1} \sigma^{3}(T-t)^{3 / 2}}\left[n\left(\bar{d}_{1}\right)\left(\bar{d}_{1}^{2}-\sigma^{2}(T-t)-1\right)-n\left(\bar{d}_{2}\right)\left(\bar{d}_{2}^{2}-\sigma^{2}(T-t)-1\right)\right]
\end{gathered}
$$

According to Result 2 in section 1, the solution of the first-order approximation $\sqrt{\varepsilon} V_{1}^{s}(t, s)$ is explicitly given by the following:

$$
\begin{aligned}
\sqrt{\varepsilon} V_{1}^{s}(t, s) & =-(T-t)\left(C_{1} s^{2} \frac{\partial^{2} V_{0}^{s}}{\partial s^{2}}+C_{2} s^{3} \frac{\partial^{3} V_{0}^{s}}{\partial s^{3}}\right) \\
& =\left\{\begin{array}{l}
\frac{-C_{1} s}{K_{1} \bar{\sigma}}\left[n\left(\bar{d}_{1}\right)-n\left(\bar{d}_{2}\right)\right]+\frac{-C_{1} s}{K_{1} \bar{\sigma}^{2} \sqrt{T-t}}\left[(-\bar{d}) n\left(\bar{d}_{1}\right)+\left(\bar{d}_{2}\right) n\left(\bar{d}_{2}\right)\right] \\
+\frac{-C_{2} s}{K_{1} \bar{\sigma}^{3}(T-t)}\left[n\left(\bar{d}_{1}\right)\left(\bar{d}_{1}^{2}-\bar{\sigma}^{2}(T-t)-1\right)-n\left(\bar{d}_{2}\right)\left(\bar{d}_{2}^{2}-\bar{\sigma}^{2}(T-t)-1\right)\right]
\end{array}\right\}
\end{aligned}
$$

Combining the result of (12) and (13) we obtain the approximate price $\tilde{V}^{s}=V_{0}^{s}+\sqrt{\varepsilon} V_{1}^{s}$ for the price of the supershare option under fast mean-reverting stochastic model. 


\subsection{European Chooser Option}

A chooser option is a contract in which the holder of this contract has the right to choose at some future date $T_{0}$ whether the option is to be a put or a call option with the same strike price $K$, and the expiration time $T>T_{0}$. The payoff at $T_{0}$ of a chooser option is:

$$
\begin{aligned}
& C H_{T_{0}}=\max \left[c\left(T-T_{0}, S_{T_{0}}, K\right),\right. \\
& \left.p\left(T-T_{0}, S_{T_{0}}, K\right)\right]
\end{aligned}
$$

$c(\tau, s, K)$ and $p(\tau, s, K)$ are the vanilla call option and put option respectively, these functions are given by the following formula:

$$
\begin{aligned}
& c(\tau, s, K)=s N\left(d_{1}\right)-K e^{-r \tau} N\left(d_{2}\right) \\
& p(\tau, s, K)=K e^{-r \tau} N\left(-d_{2}\right)-s N\left(-d_{1}\right) \\
& d_{1,2}=\frac{\ln (s / K)+\left(r \pm \frac{1}{2} \sigma^{2}\right) \tau}{\sigma \sqrt{\tau}}
\end{aligned}
$$

where $r, \sigma, K$ are constants, $\tau=T-t$ is time to expiry.

In the fast mean-reverting stochastic volatility economy, the approximate price of the chooser option is:

$$
\tilde{V}^{c}(t, s)=V_{0}^{c}(t, s)+\sqrt{\varepsilon} V_{1}^{c}(t, s)
$$

The first-order approximation $V_{0}^{c}(t, s)$ is simply the standard Black-Scholes option price with a constant volatility $\bar{\sigma}$.

Lemma 2 The price of the chooser option in the Black-Scholes model with constant volatility is given by:

$$
\begin{gathered}
V_{0}^{c}(t, s)=s\left(N\left(d_{1}\right)-N\left(-d_{1}^{*}\right)\right)+ \\
+K e^{-r(T-t)}\left(N\left(-d_{2}^{*}\right)-N\left(d_{2}\right)\right) \\
d_{1,2}=\frac{\ln (s / K)+\left(r \pm \frac{1}{2} \sigma^{2}\right)(T-t)}{\sigma \sqrt{T-t}} ; \\
d_{1,2}^{*}=\frac{\ln (s / K)+\left(r \pm \frac{1}{2} \sigma^{2}\right)\left(T_{0}-t\right)}{\sigma \sqrt{T_{0}-t}}
\end{gathered}
$$

Where $K, r, \sigma, T_{0}<T$ are constants. $S_{t}$ is the underlying asset price at time $t, T$ is the expiry date.

\section{Proof of Lemma2:}

The payoff at $T_{0}$ of a chooser option is:

$$
C H_{T_{0}}=\max \left[c\left(T-T_{0}, S_{T_{0}}, K\right), p\left(T-T_{0}, S_{T_{0}}, K\right)\right]
$$

According to the call-put parity, a put option at time $T_{0}$ can be written as:

$$
\begin{aligned}
& p\left(T-T_{0}, S_{T_{0}}, K\right)=c\left(T-T_{0}, S_{T_{0}}, K\right)- \\
& -S_{T_{0}}+K e^{-r\left(T-T_{0}\right)}
\end{aligned}
$$

thus the payoff of a chooser option at time $T_{0}$ can be rewritten as:

$$
\begin{aligned}
& C H_{T_{0}}=\max \left[c\left(T-T_{0}, S_{T_{0}}, K\right), c\left(T-T_{0}, S_{T_{0}}, K\right)-\right. \\
& \left.-S_{T_{0}}+K e^{-r\left(T-T_{0}\right)}\right]
\end{aligned}
$$

The payoff above is equivalent to:

$$
C H_{T_{0}}=c\left(T-T_{0}, S_{T_{0}}, K\right)+\left(K e^{-r\left(T-T_{0}\right)}-S_{T_{0}}\right)^{+}
$$

which implies the standard chooser option is equivalent to a portfolio composed of a long call option with strike $K$, expiration time $T$ and a long put with strike $K e^{-r\left(T-T_{0}\right)}$, expiration time $T_{0}$. So the arbitrage price of a standard chooser option at time $t \in\left[0, T_{0}\right]$ is:

$$
\begin{aligned}
& \operatorname{ch}\left(t, S_{t}\right)=C H_{t}=c\left(T-t, S_{t}, K\right)+ \\
& +p\left(T_{0}-t, S_{t}, K e^{-r\left(T-T_{0}\right)}\right)
\end{aligned}
$$

Using the vanilla call and put option formula, we have:

$$
\begin{aligned}
& \operatorname{ch}(t, s)=s N\left(d_{1}\right)-K e^{-r(T-t)} N\left(d_{2}\right)+ \\
& +K e^{-r\left(T-T_{0}\right)} e^{-r\left(T_{0}-t\right)} N\left(-d_{2}^{*}\right)-s N\left(-d_{1}^{*}\right) \\
& =s\left(N\left(d_{1}\right)-N\left(-d_{1}^{*}\right)\right)+K e^{-r(T-t)}\left(N\left(-d_{2}^{*}\right)-N\left(d_{2}\right)\right)
\end{aligned}
$$

where

$$
d_{1,2}=\frac{\ln (s / K)+\left(r \pm \frac{1}{2} \sigma^{2}\right)(T-t)}{\sigma \sqrt{T-t}} \text { and }
$$




$$
d_{1,2}^{*}=\frac{\ln (s / K)+\left(r \pm \frac{1}{2} \sigma^{2}\right)\left(T_{0}-t\right)}{\sigma \sqrt{T_{0}-t}}
$$

Hence according to Result 1 in section 2 and lemma $2, V_{0}^{c}(t, s)$ is given by:

$$
\begin{aligned}
& V_{0}^{c}(t, s)=s\left(N\left(d_{1}\right)-N\left(-d_{1}^{*}\right)\right)+ \\
& +K e^{-r(T-t)}\left(N\left(-d_{2}^{*}\right)-N\left(d_{2}\right)\right) \\
& d_{1,2}=\frac{\ln (s / K)+\left(r \pm \frac{1}{2} \bar{\sigma}^{2}\right)(T-t)}{\bar{\sigma} \sqrt{T-t}} ; \\
& d_{1,2}^{*}=\frac{\ln (s / K)+\left(r \pm \frac{1}{2} \bar{\sigma}^{2}\right)\left(T_{0}-t\right)}{\bar{\sigma} \sqrt{T_{0}-t}}
\end{aligned}
$$

From the definition of $d_{1,2}$ and $d_{1,2}^{*}$, we have $\frac{\partial d_{1,2}}{\partial s}=\frac{1}{s \bar{\sigma} \sqrt{T-t}}$ and $\frac{\partial d_{1,2}^{*}}{\partial s}=\frac{1}{s \bar{\sigma} \sqrt{T_{0}-t}}$. Hence, the second order and third order derivative of the value of the zero-order approximation with respect to the underlying asset price are given by:

$$
\begin{aligned}
& \frac{\partial^{2} V_{0}^{c}}{\partial s^{2}}=\frac{n\left(d_{1}\right)}{s \bar{\sigma} \sqrt{T-t}}+\frac{n\left(d_{1}^{*}\right)}{s \bar{\sigma} \sqrt{T_{0}-t}} \\
& \frac{\partial^{3} V_{0}^{c}}{\partial s^{3}}=\frac{-n\left(d_{1}\right)}{s^{2} \bar{\sigma} \sqrt{T-t}}\left(\frac{d_{1}}{\bar{\sigma} \sqrt{T-t}}+1\right)+ \\
& +\frac{-n\left(d_{1}^{*}\right)}{s^{2} \bar{\sigma} \sqrt{T_{0}-t}}\left(\frac{d_{1}^{*}}{\bar{\sigma} \sqrt{T_{0}-t}}+1\right)
\end{aligned}
$$

According to the Results 2 in Section 1, the solution of the first-order approximation $\sqrt{\varepsilon} V_{1}^{c}(t, s)$ is explicitly given by the following:

$$
\begin{aligned}
& \sqrt{\varepsilon} V_{1}^{c}(t, s)=-(T-t)\left(C_{1} s^{2} \frac{\partial^{2} V_{0}^{c}}{\partial s^{2}}+C_{2} s^{3} \frac{\partial^{3} V_{0}^{c}}{\partial s^{3}}\right) \\
& \left.=-(T-t)\left\{\begin{array}{l}
\frac{C_{1} s}{\bar{\sigma}}\left[\frac{n\left(d_{1}\right)}{\sqrt{T-t}}+\frac{n\left(d_{1}^{*}\right)}{\sqrt{T_{0}-t}}\right] \\
+\frac{-C_{2} s}{\bar{\sigma}}\left[\begin{array}{l}
\frac{n\left(d_{1}\right)}{\sqrt{T-t}}\left(\frac{d_{1}}{\bar{\sigma} \sqrt{T-t}}+1\right) \\
+\frac{n\left(d_{1}^{*}\right)}{\sqrt{T_{0}-t}}\left(\frac{d_{1}^{*}}{\bar{\sigma} \sqrt{T_{0}-t}}+1\right.
\end{array}\right)
\end{array}\right)\right\}
\end{aligned}
$$

Combining the result of (15) and (16) we obtain the approximate price $\tilde{V}^{c}=V_{0}^{c}+\sqrt{\varepsilon} V_{1}^{c}$ for the price of the chooser option under fast mean-reverting stochastic model.

\section{Results and Discussion}

From the result of Section 2, an approximation for the price of supershare option and chooser option under fast mean-reverting stochastic volatility economy has been formally obtained, and the parameters in the stochastic volatility model are reduced to $C_{1}$ and $C_{2}$. These two parameters can be calibrated from implied volatility surface. In order to illustrate with a numerical example, we consider a supershare option with the strike prices of $K_{1}=400$ and $K_{2}=500$; we also consider a chooser option with strike price $K=460$ and $T_{0}=0.5$. Following parameters are chosen throughout the numerical computation: the (constant) effective volatility $\bar{\sigma}=0.1$; the time to maturity $T-t=1$; the risk-free interest rate $r=2 \%$. the underlying asset price at time 0 is chosen as $s \in[400,500]$; two calibrated parameters are $C_{1}=-0.0044$ and $C_{2}=0.000154$ respectively.

The numerical result of the zero-order approximation (B-S price) and approximation price of the supershare option price at time 0 is shown in Fig. 1; Fig. 2 shows the ratio of the firstorder approximation and the approximation price of the supershare option.

From the numerical results of the supershare option, one can observe that ratio of the firstorder approximation and the approximation price is always negative, hence the supershare option under the fast mean-reverting stochastic volatility is underpriced compared to its B-S price which can be observed in Fig. 1; Fig. 2 shows the ratio of the first-order approximation and the approximation price of the supershare option.

The numerical result of the zero-order approximation (B-S price) and approximation price of the chooser option price at time 0 is shown in Fig. 3; Fig. 4 shows the ratio of the firstorder approximation and the approximation price of the chooser option.

From the numerical results of the chooser option, one can observe that ratio of the firstorder approximation and the approximation price is always positive, hence the price of the chooser option under the fast meanreverting stochastic volatility is higher than its B-S price which can be observed in Fig. 3 . 
The zero-order approximation price (solid) and the stochastic volatility approximation price (dashed) for supershare options

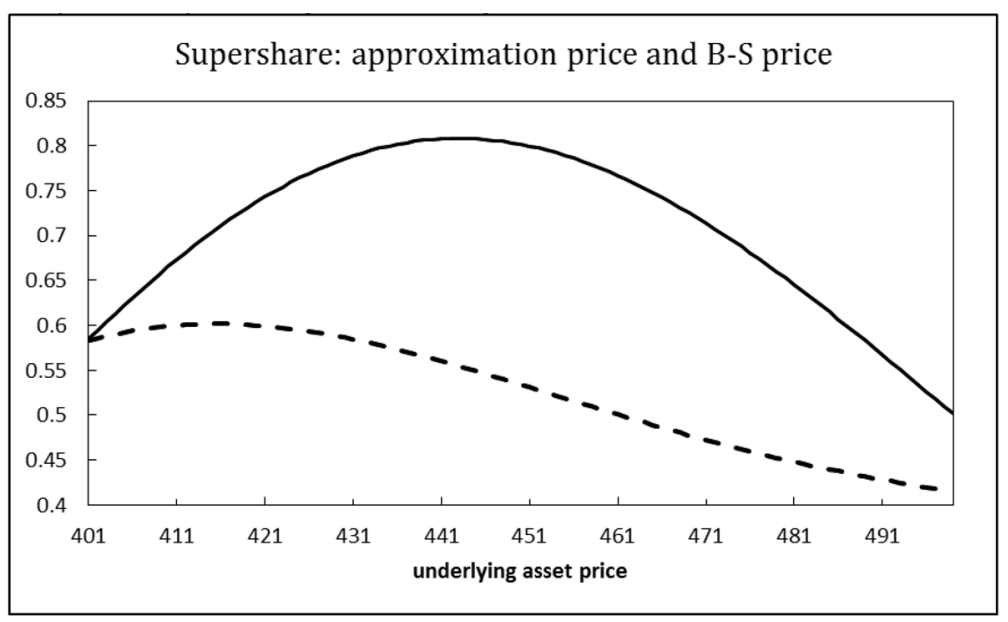

The ratio of the first-order approximation and the approximation price for supershare options

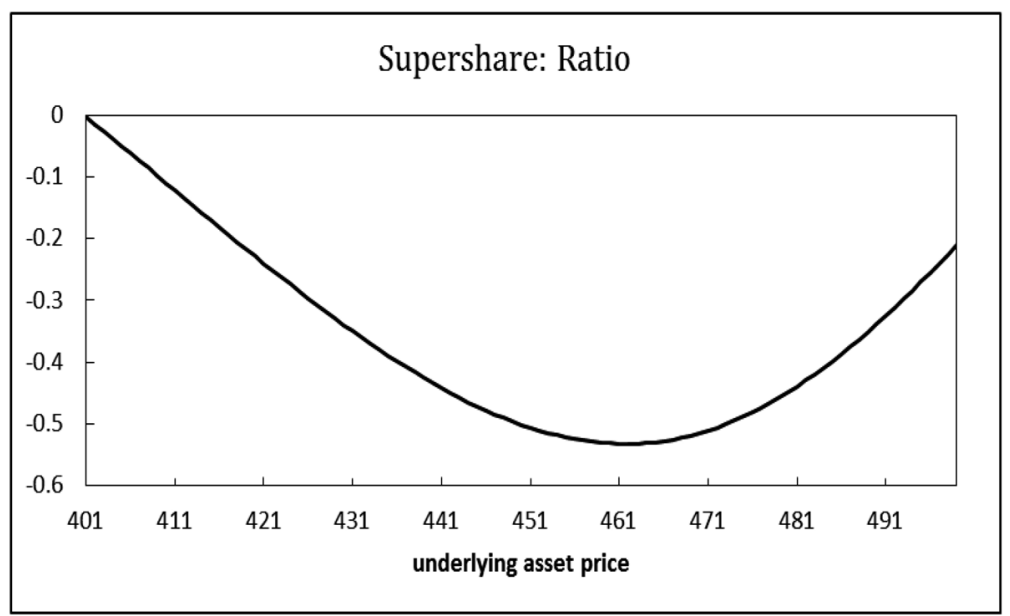



approximation price (dashed) for chooser options
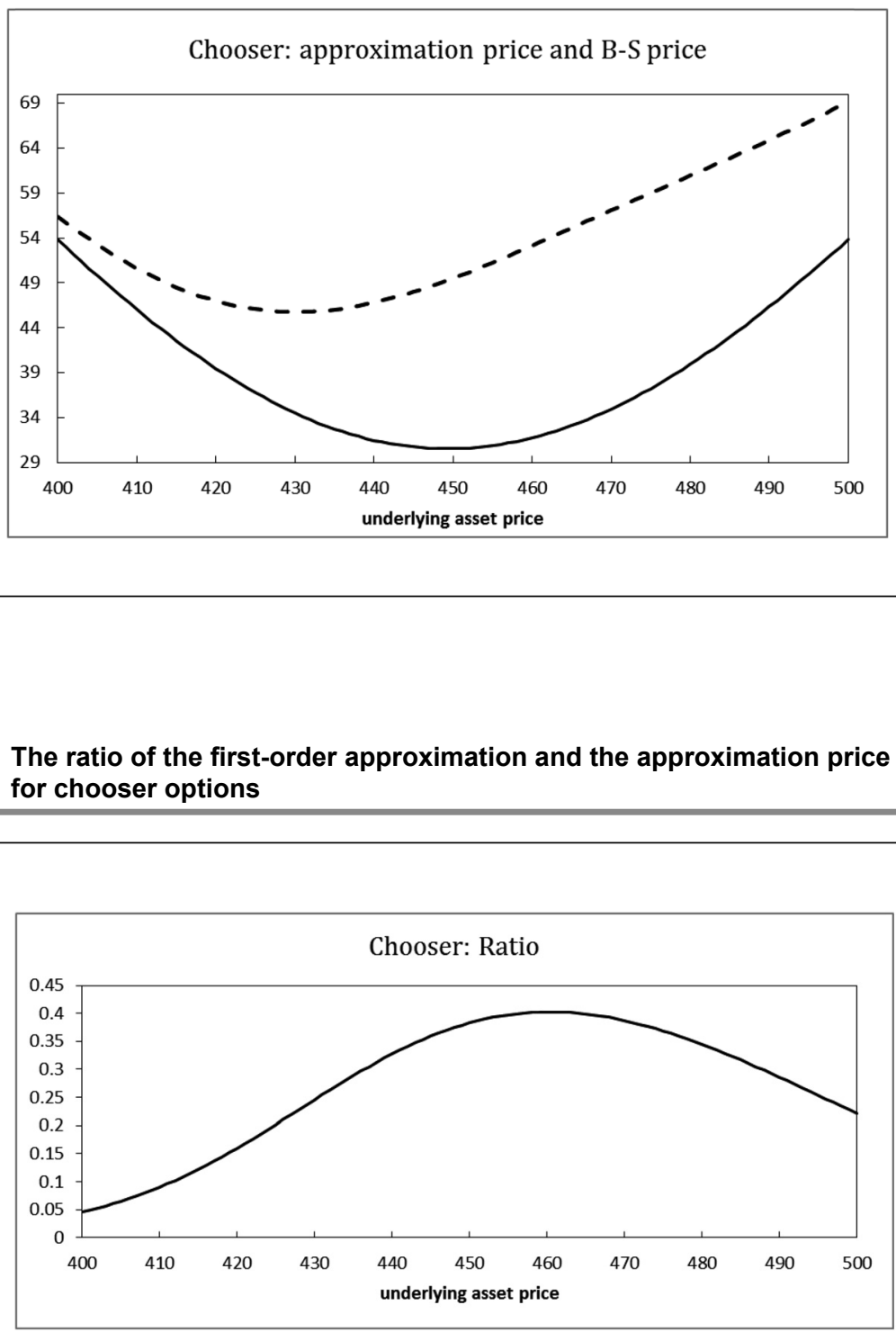

Source: own 


\section{Conclusions}

In this paper, we derive the zero-order approximation term and the first-order approximation term for the suppershare option and chooser option under a fast meanreverting stochastic volatility scenario. By deriving the approximation term, we obtained the analytic approximation formulae for these two European exotic option under a stochastic volatility model. The numerical analysis in our model shows that stochastic volatility of underlying asset underprices the supershare options, while in the case of the chooser options its price in stochastic volatility model is higher than the price in the constant volatility model. Further studies will be carried out on other type of exotic options and calibrating the parameters from the implied volatility surface will be our subsequent research.

Guangdong Provincial Humanities and Social Science Platform-Pearl River Delta Industrial Ecology Research Center (2016WZJD005).

Guangdong Social Science Research Base - Quality and Brand Development and Research Center of Dongguan University of Technology (GB200101).

"Supported by Research start-up funds of DGUT (GC300501-148)".

\section{References}

Alizaden, S., Brandt, M., \& Diebold, F. (2002). Range-based estimation of stochastic volatility models. Journal of Finance, 57(3), 1047-1091. 6261.00454.

Bernales, A., \& Guidolin, M. (2015). Learning to smile: Can rational learning explain predictable dynamics in the implied volatility surface. Journal of Financial Markets, 26(1), 1-37. https://doi.org/10.1016/j. finmar.2015.10.002.

Cao, J. L., Lian, G. H., \& Roslan, T. (2016). Pricing variance swaps under stochastic volatility and stochastic interest rate. Applied Mathematics and Computation, 277(20), 72-81. http://dx.doi.org/10.1016/j.amc.2015.12.027.
Carmichael, B., \& Coen, A. (2013). Asset pricing with skewed-normal return. Finance Research Letters, 10(2), 50-57. http://dx.doi. org/10.1016/j.frl.2013.01.001.

Fouque, J. P., Papanicolaou, G., Sircar, R., \& Solna, K. (2003). Singular perturbations in option pricing. SIAM Journal on Applied Mathematics, 63(5), 1648-1665. http://dx.doi. org/10.1137/S0036139902401550.

Heston, S. L. (1993). A closed-form solution for options with stochastic volatility with applications to bond and currency options. The Review of Financial Studies, 6(2), 327-343. http://dx.doi.org/10.1093/rfs/6.2.327.

Lee, M. K., Yang, S. J., \& Kim, J. H. (2016). A closed form solution for vulnerable options with Heston's stochastic volatility. Chaos, Solitons \& Fractals, 86(1), 23-27. http://dx.doi. org/10.1016/j.chaos.2016.01.026.

Park, S. H., \& Kim, J. H. (2013). A semianalytic pricing formula for lookback options under a general stochastic volatility model. Statistics \& Probability Letters, 83(11), 2537-2543. http:// dx.doi.org/10.1016/j.spl.2013.08.002.

Smith, G. P. (2015). New Evidence on Sources of Leverage Effects in Individual Stocks. Financial Review, 50(3), 331-340. http://dx.doi.org/10.1111/fire.12069.

Wong, H. Y., \& Chan, C. M. (2008). Turbo warrants under stochastic volatility. Quantitative Finance, 8(7), 739-751. http://dx.doi. org/10.1080/14697680701691469.

Yang, S. J., Lee, M. K., \& Kim, J. H. (2014). Pricing vulnerable options under a stochastic volatility model. Applied Mathematics Letters, 34(1), 7-12. http://dx.doi.org/10.1016/j.aml.2014.03.007.

Zhu, S. P., \& Lian, G. H. (2015). Pricing forward-start variance swaps with stochastic volatility. Applied Mathematics and Computation, 250(1), 920-933. http://dx.doi. org/10.1016/j.amc.2014.10.050.

Pengshi Li, Ph.D. Dong Guan University of Technology School of Economics and Management Department of Accounting and Finance China 2018074@dgut.edu.cn 


\section{Abstract}

\section{PRICING EXOTIC OPTION UNDER STOCHASTIC VOLATILITY MODEL}

\section{Pengshi Li}

This paper studies supershare and chooser options in a stochastic volatility economy. These two options are typical exotic options which suggest a broad range of usage and application in different financial market conditions. Despite the popularity and longevity of the Black-Scholes model, the assumption of constant volatility in the Black-Scholes model contradicts to the existence of the non-flat implied volatility surface observed in empirical studies. Although many studies are devoted to option pricing under stochastic volatility model in recent years, to the best of our knowledge, research on exotic option such as supershare and chooser option pricing have not been carried out in the stochastic volatility case. Supershare and chooser options are both important financial instruments, research on these two exotic options in stochastic volatility model may give more insights on the pricing of supershare and chooser options. By extending the constant volatility in the Black-Scholes model, this paper studies the pricing problem of the supershare option and chooser options in a fast mean-reverting stochastic volatility scenario. Analytic approximation formulae for these two exotic options in fast mean-reverting stochastic volatility model are derived according to the method of asymptotic expansion which shows the approximation option price can be expressed as the combination of the zero-order and first-order approximations. By incorporating the stochastic volatility effect, the numerical analysis in our model shows that stochastic volatility of underlying asset underprices the supershare options, while in the case of the chooser options its price in stochastic volatility model is higher than the price in the constant volatility model.

Keywords: Exotic options, supershare, chooser, stochastic volatility, mean-reverting.

JEL Classification: C40.

DOI: 10.15240/tul/001/2019-4-009 